\title{
Myasthenia gravis in patients with thymoma affects survival rate following extended thymectomy
}

\author{
ZHEFENG ZHANG $^{1 *}$, YOUBIN CUI $^{2 *}$, RUI JIA $^{1}$, LEI XUE ${ }^{1}$ and HUAGANG LIANG ${ }^{1}$ \\ ${ }^{1}$ Department of Thoracic Surgery, The First Hospital of Qinhuangdao, Qinhuangdao, Hebei 066000; \\ ${ }^{2}$ Department of Thoracic Surgery, The First Hospital of Jilin University, Changchun, Jilin 130021, P.R. China
}

Received March 6, 2015; Accepted March 18, 2016

DOI: $10.3892 / \mathrm{ol} .2016 .4528$

\begin{abstract}
Thymomas are the most common adult tumors in the anterior mediastinal compartment, and a significant amount of thymomas are complicated by myasthenia gravis (MG). Extended thymectomy (ET) is the primary treatment method for thymomas and is used to completely resect possible ectopic thymus to avoid recurrence. Studies on the effect of MG in thymoma patients following ET are limited. The aim of the present study was to determine whether the presence of $\mathrm{MG}$ affects the prognosis of patients with thymoma. The present study consisted of 104 patients with thymoma that underwent ET; 61 men $(58.7 \%)$ and 43 women (41.3\%) (mean age, 54.6 years). In total, 38 patients had MG (36.5\%). MG was most frequently observed in World Health Organization (WHO) classification type B2 thymoma compared with other types of thymoma. During the 5-year follow-up period, 11 patients succumbed to a recurrence of thymoma or respiratory failure due to MG. The overall 5-year survival rate in patients without MG or with MG was 89.1 and $76.0 \%$, respectively. The overall survival (OS) rate in patients with Masaoka stages I + II and III + IV was 90.0 and $68.0 \%$, respectively. The OS rate in patients with $\mathrm{WHO}$ type $\mathrm{A}+\mathrm{AB}+\mathrm{B} 1$ and type $\mathrm{B} 2+\mathrm{B} 3$ was 96.9 and $76.8 \%$, respectively. The patients with $\mathrm{MG}(\mathrm{P}=0.026)$, Masaoka stages III + IV $(\mathrm{P}=0.008)$ and WHO type $\mathrm{B} 2+\mathrm{B} 3$ $(\mathrm{P}=0.032)$ had a poorer prognosis compared with patients without these characteristics. Furthermore, multivariate analysis by Cox regression revealed that age $[\mathrm{P}=0.032$; relative risk $(\mathrm{RR})=1.097 ; 95 \%$ confidence interval $(\mathrm{CI})=1.097-1.192]$
\end{abstract}

Correspondence to: Dr Huagang Liang, Department of Thoracic Surgery, The First Hospital of Qinhuangdao, 258 Wenhua Road, Qinhuangdao, Hebei 066000, P.R. China

E-mail: 13315397676@163.com

*Contributed equally

Abbreviations: MG, myasthenia gravis; ET, extended thymectomy; WHO, World Health Organization; VATS, video-assisted thoracoscopic surgery; OS, overall survival

Key words: thymoma, myasthenia gravis, extended thymectomy, survival analysis and MG $(\mathrm{P}=0.042 ; \mathrm{RR}=0.167 ; 95 \% \mathrm{CI}=0.037-0.940)$ significantly affected OS rate. In summary, ET is a reliable method for the treatment of thymoma. Long-term survival is expected for patients at early Masaoka stages, and for patients without MG. The prognosis of patients with thymomas with MG is poorer compared with patients without MG. The present findings provide useful information for the future management of patients with thymomas.

\section{Introduction}

Thymoma is a tumor originating from the epithelial cells of the thymus and is a relatively rare neoplasm with an incidence of 0.13 cases per 100,000 individuals per year $(1,2)$. The prognosis of patients with thymoma largely depends on the Masaoka stage of disease and prognosis is poorer for patients with stage III or IV compared with patients with stage I and II tumors (3). The 10-year survival rates are 90, 70, 55 and 35\% for stages I, II, III, and IV thymoma, respectively (4). Surgical resection is the primary treatment method for thymoma and a sternotomy is the optimal surgical procedure for thymoma, since it may not be possible to perform a complete thymectomy via thoracotomy on locally advanced lesions, particularly those that are stage III-IV $(5,6)$. Normally, thymoma is a slow-growing tumor, but $40 \%$ of thymomas exhibit a locally invasive growth pattern. In addition, thymomas often result in the development of pleural dissemination and distant metastasis $(7,8)$. Therefore, complete resection should be the primary goal during surgical treatment. The International Thymic Malignancies Interest Group recommends en bloc resection, also known as extended thymectomy (ET), including complete thymectomy and resection of the surrounding mediastinal fat, due to the possibility of macroscopically invisible invasion of the tumor (5). Due to the limited case number of thymomas, survival analysis following complete thymectomy based on large cohorts is limited. Therefore, gaining an improved understanding of survival prognosis may be beneficial for the management of the patients with thymoma.

Aside from disease stage, other factors that affect the outcome of patients following complete thymectomy remain largely unknown. Thymoma is frequently associated with parathymic diseases, including myasthenia gravis (MG) (9). MG is the most commonly associated paraneoplastic disease in thymoma patients and $30-50 \%$ of thymoma patients have 
MG (10). However, whether MG is a determining factor for the outcome of patients with thymoma following complete thymectomy remains unknown. The present study demonstrated that the prognosis of patients with thymomas and MG is poorer compared with patients without MG. Therefore, MG status should be considered in the management of patients with thymomas.

\section{Materials and methods}

Patients. In total, 104 patients with thymoma were consecutively recruited to the present study between January 2005 and January 2010. All the patients were recruited at two centers (The First Hospital of Qinhuangdao, Qinhuangdao; the First Hospital of Jilin University, Changchun, China). The clinical characteristics of the patients were recorded, including gender, age, presence of MG, computed tomography (CT) of the chest, World Health Organization (WHO) type, Masaoka stage, myasthenic crisis presence and surgical treatment procedure. Follow-up was performed for all patients every 3 months through office visits or telephone interviews and the follow-up information consisted of postoperative MG status and physical examination. In addition, chest roentgenography and chest CT scans were performed every 6 months for the first 2 years following surgery and annually thereafter. The present study was approved by The First Hospital of Qinhuangdao Ethics Committee and all patients provided signed informed consent prior to participation.

Clinical and histological staging. The clinical stage of the tumors was evaluated according to the Masaoka staging system classification (11): Stage I, completely encapsulated and lacking microscopic capsular invasion; Stage II, microscopic capsular invasion or macroscopic invasion into mediastinal pleura or surrounding fatty tissue; stage III, macroscopic invasion into adjacent organs; stage IV, tumor with pleural or pericardial dissemination (IVa) or hematogenous or lymphogenous distant metastasis (IVb). The histological subtype of thymoma was defined according to the 2004 WHO histological classification (12). The WHO classification system categorizes tumors as follows: Type A, comprised of a homogenous population of neoplastic epithelial cells with spindle/oval shape, lacking nuclear atypia, and accompanied by few or no non-neoplastic lymphocytes; type AB, foci possessing features of type A thymoma are admixed with foci rich in lymphocytes, the segregation of two patterns can be sharp or indistinct; type B1, resembles the normal functional thymus in that it combines large expanses with an appearance practically indistinguishable from that of normal thymic cortex, with areas resembling the thymic medulla; type B2, the neoplastic epithelial component appears as scattered, plump cells with vesicular nuclei and distinct nucleoli among a heavy population of lymphocytes, and perivascular spaces are common; type B3, comprised predominantly of epithelial cells with a round or polygonal shape that exhibit mild atypia admixed with a minor component of lymphocytes, and foci of squamous metaplasia and perivascular spaces are common. Masaoka stage and WHO classification were confirmed following histological examination of hematoxylin and eosin-stained sections $(5 \mu \mathrm{m})$ derived from paraffin embedded blocks.
Surgical procedures. Two surgical procedures were used: Median sternotomy and video-assisted thoracoscopic surgery (VATS). ET was defined as the resection of the entire thymus and mediastinal fat tissue between the two phrenic nerves. For Masaoka stages III and IV, combined organ and tissue resection was required and performed.

Survival analysis. The date of surgery was considered at the time of diagnosis. The survival durations were calculated between the date of surgery and the date of death due to any cause or the last follow-up day in January 2015. Overall survival (OS) time was defined as the time between the date of diagnosis to date of death due to any cause.

Statistical analysis. The Kaplan-Meier method was used to estimate the probability of survival and the differences between survival in each group was analyzed by the log-rank test. Categorical variables were compared using the $\chi^{2}$-test or Fisher's exact test. Multivariate analysis was performed using a Cox regression model. Statistical analysis was performed using SPSS version 17.0 (SPSS, Inc., Chicago, IL, USA). $\mathrm{P}<0.05$ was considered to indicate a statistically significant difference.

\section{Results}

Patients characteristics. Out of the 104 patients, 63 were men and 41 were women. The mean age was 54.6 years (range, 20-79 years). As shown in Table I, all patients were divided into two groups based on whether or not the patient presented with MG. In addition, 30 patients presented with coronary disease, hypertension or diabetes. In total, 8 patients $(7.7 \%)$ were WHO type A, 22 (21.2\%) were AB, 17 (16.3\%) were B1, $34(32.7 \%)$ were B2 and 23 (22.1) were B3. According to the Masaoka staging system, 46 patients $(44.2 \%)$ were diagnosed with stage I disease, 42 patients $(40.4 \%)$ had stage II disease, 14 patients $(13.5 \%)$ had stage III disease and 2 patients $(1.9 \%)$ had stage IVa disease. In total, 38 patients $(36.5 \%)$ had MG. In a comparison between the presence and absence of $\mathrm{MG}$, the prevalence of MG was significantly higher in type B2 thymoma compared with other types of thymoma $(\mathrm{P}=0.019)$.

Masaoka staging and WHO classification. The association between Masaoka stage and WHO classification was analyzed and is presented in Table II. The percentage of patients with Masaoka stages I, II, III and IVa was 42.3, 39.4, 16.3 and $1.9 \%$, respectively. The percentage of patients with WHO type A, AB, B1, B2 and B3 was 7.7, 21.2, 16.3, 32.7 and $22.1 \%$, respectively. The majority of patients with WHO type A, AB and B1 was observed at Masaoka stage I and II (44/47; 93.6\%). However, thymomas classified as WHO type B2 and B3 subtype $(16 / 19 ; 84.2 \%)$, which corresponded to Masaoka stage III and IVa, exhibited more invasive behavior and thus were considered more aggressive. The association between tumor size and Masaoka stage is indicated in Fig. 1A. The mean tumor size with Masaoka stages I and II was significantly smaller compared with tumors with Masaoka stages III and IV $(\mathrm{P}<0.001)$. No significant association was observed between tumor size and WHO classification $(\mathrm{P}=0.488)$ (Fig. 1B) 
Table I. Characteristics of 104 thymoma patients with and without MG.

\begin{tabular}{|c|c|c|c|c|c|}
\hline Characteristic & Total & Without MG & With MG & $\mathrm{t} / \chi^{2}$ value & P-value \\
\hline Total patients, $\mathrm{n}$ & $104(100)$ & $66(64)$ & $38(37)$ & & \\
\hline Age at surgery, years & $54.6 \pm 11.0$ & $56.0 \pm 10.8$ & $52.2 \pm 11.1$ & 1.714 & 0.090 \\
\hline Gender, n (\%) & & & & 1.849 & 0.174 \\
\hline Male & $61(59)$ & $42(64)$ & $19(50)$ & & \\
\hline Female & $43(41)$ & $24(36)$ & $19(50)$ & & \\
\hline Tumor size, $\mathrm{cm}$ & $3.1 \pm 1.3$ & $3.2 \pm 1.4$ & $3.0 \pm 0.9$ & 0.610 & 0.543 \\
\hline Surgical procedure, $\mathrm{n}(\%)$ & & & & 0.865 & 0.352 \\
\hline Sternotomy & $10(10)$ & $5(8)$ & $5(13)$ & & \\
\hline VATS & $94(90)$ & $61(92)$ & $33(87)$ & & \\
\hline Myasthenic crisis, n (\%) & $8(8)$ & $0(0)$ & $8(21)$ & 7.538 & $0.006^{\mathrm{b}}$ \\
\hline WHO histological classification, n (\%) & & & & 11.83 & $0.019^{\mathrm{a}}$ \\
\hline A & $8(8)$ & $7(11)$ & $1(3)$ & 4.500 & $0.034^{\mathrm{a}}$ \\
\hline $\mathrm{AB}$ & $22(21)$ & $17(26)$ & $5(13)$ & 6.545 & $0.011^{\mathrm{a}}$ \\
\hline B1 & $17(16)$ & $12(18)$ & $5(13)$ & 2.882 & 0.090 \\
\hline $\mathrm{B} 2$ & $34(33)$ & $14(21)$ & $20(53)^{\mathrm{c}}$ & 0.471 & 0.493 \\
\hline B3 & $23(22)$ & $16(24)$ & $7(18)$ & 2.130 & 0.144 \\
\hline Masaoka stage, n (\%) & & & & 3.962 & 0.266 \\
\hline I & $46(44)$ & $30(45)$ & $16(42)$ & 6.712 & $0.010^{\mathrm{a}}$ \\
\hline II & $42(40)$ & $28(42)$ & $14(37)$ & 4.677 & $0.031^{\mathrm{a}}$ \\
\hline III & $14(14)$ & $6(9)$ & $8(21)$ & 0.059 & 0.808 \\
\hline IVa & $2(2)$ & $2(3)$ & $0(0)$ & & \\
\hline Pathology of paraneoplastic thymus, n (\%) & & & & 13.975 & $<0.001^{\mathrm{b}}$ \\
\hline Involuted & $87(84)$ & $62(94)$ & $25(66)$ & & \\
\hline Hyperplastic & $17(16)$ & $4(6)$ & $13(34)$ & & $<0.001^{\mathrm{b}}$ \\
\hline Radiotherapy, n (\%) & $49(47)$ & $25(38)$ & $24(63)$ & 6.185 & $0.013^{\mathrm{a}}$ \\
\hline Recurrence, n (\%) & $3(3)$ & $1(2)$ & $2(5)$ & 92.346 & $<0.001^{\mathrm{b}}$ \\
\hline Mortality, n (\%) & $11(11)$ & $3(5)$ & $8(21)$ & 9.013 & $0.003^{\mathrm{b}}$ \\
\hline
\end{tabular}

${ }^{\mathrm{a}} \mathrm{P}<0.05 .{ }^{\mathrm{b}} \mathrm{P}<0.01 .{ }^{\mathrm{c}} \mathrm{Prevalence}$ of $\mathrm{MG}$ was significantly higher in type $\mathrm{B} 2$ thymoma compared with other types of thymoma. MG, myasthenia gravis; VATS, video-assisted thoracoscopic surgery; WHO, World Health Organization.

Table II. Association between Masaoka stage and WHO histological classification.

WHO histological classification, $\mathrm{n}$

\begin{tabular}{|c|c|c|c|c|c|c|c|}
\hline \multirow[b]{2}{*}{ Masaoka stage, $\mathrm{n}$} & \multirow[b]{2}{*}{ Total patients, $\mathrm{n}$} & \\
\hline & & A & $\mathrm{AB}$ & B1 & $\mathrm{B} 2$ & B3 & Mortalities \\
\hline Total & 104 & 8 & 22 & 17 & 34 & 23 & 11 \\
\hline I & 46 & 4 & 9 & 6 & 17 & 8 & 1 \\
\hline II & 42 & 4 & 11 & 10 & 6 & 10 & 3 \\
\hline III & 14 & 0 & 2 & 1 & 10 & 4 & 6 \\
\hline IVa & 2 & 0 & 0 & 0 & 1 & 1 & 1 \\
\hline Mortalities & 11 & 0 & 1 & 0 & 5 & 5 & - \\
\hline
\end{tabular}

WHO, World Health Organization.

Surgical procedure and mortalities. All patients underwent ET either by sternotomy (10 patients) or VATS (94 patients). In addition, combined resection with adjacent organs, including the lung $(n=6)$, pericardium $(n=5)$, left brachiocephalic $(n=3)$ 
Table III. Univariate and multivariate analysis for patients that underwent extended thymectomy for thymoma treatment.

\begin{tabular}{|c|c|c|c|c|c|c|}
\hline \multirow[b]{2}{*}{ Characteristic } & \multicolumn{3}{|c|}{ Univariate analysis } & \multicolumn{3}{|c|}{ Multivariate analysis } \\
\hline & P-value & $\mathrm{RR}$ & $95 \% \mathrm{CI}$ & P-value & $\mathrm{RR}$ & $95 \% \mathrm{CI}$ \\
\hline Gender & 0.299 & 2.020 & $0.536-7.619$ & 0.512 & - & - \\
\hline $\mathrm{Age}^{\mathrm{a}}$ & 0.001 & 1.138 & $1.055-1.228$ & 0.032 & 1.097 & $1.010-1.192$ \\
\hline Tumor size ${ }^{b}$ & $<0.001$ & 2.288 & $1.491-3.510$ & 0.052 & - & - \\
\hline MG association & 0.042 & 3.974 & $1.053-14.991$ & 0.042 & 0.167 & $0.037-0.940$ \\
\hline WHO classification ${ }^{\mathrm{c}}$ & 0.046 & 2.054 & $1.011-4.172$ & 0.084 & - & - \\
\hline Masaoka stage $^{\mathrm{d}}$ & 0.009 & 2.400 & $1.239-4.648$ & 0.541 & - & - \\
\hline Surgical procedure & 0.003 & 0.145 & $0.041-0.510$ & 0.574 & - & - \\
\hline Radiotherapy & 0.026 & 0.175 & $0.038-0.814$ & 0.322 & - & - \\
\hline
\end{tabular}

${ }^{\mathrm{a}}<60$ vs. $\geq 60$ years. ${ }^{\mathrm{b}}<3$ vs. $\geq 3 \mathrm{~cm}$. ${ }^{\mathrm{c}}$ Stage A vs. AB vs. B1 vs. B2 vs. B3. ${ }^{\mathrm{d}}$ Stage I vs. II vs. III vs. IVa. ${ }^{\mathrm{e}}$ Sternotomy vs. video-assisted thoracoscopic surgery. WHO, World Health Organization; CI, confidence interval; RR, relative risk; Y, yes; N, no.

A

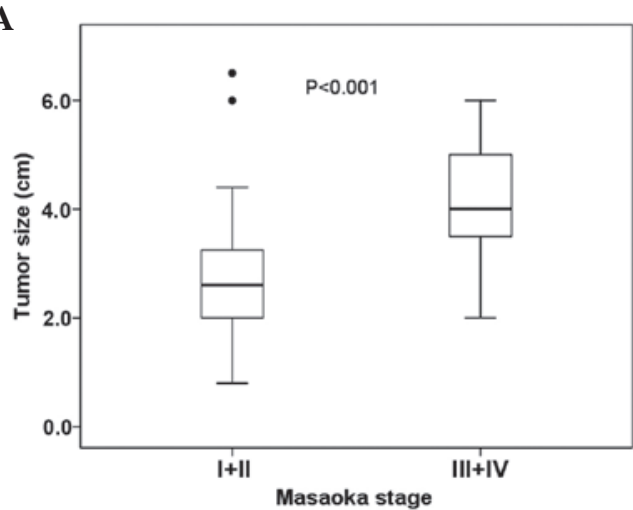

B

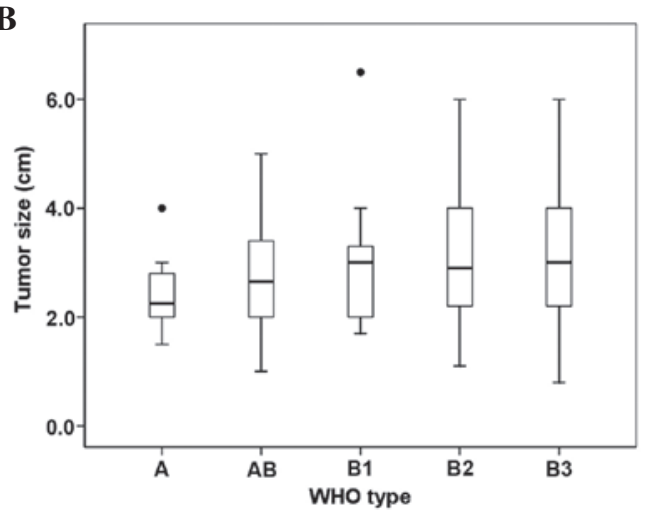

Figure 1. Tumor size divided according to Masaoka stage or WHO histological classification. (A) Association between tumor size and Masaoka stage. The tumor size in Masaoka stage I and II was significantly smaller compared with stages III and IV (P<0.001). (B) Association between tumor size and WHO histological classification. WHO, World Health Organization.

A



B

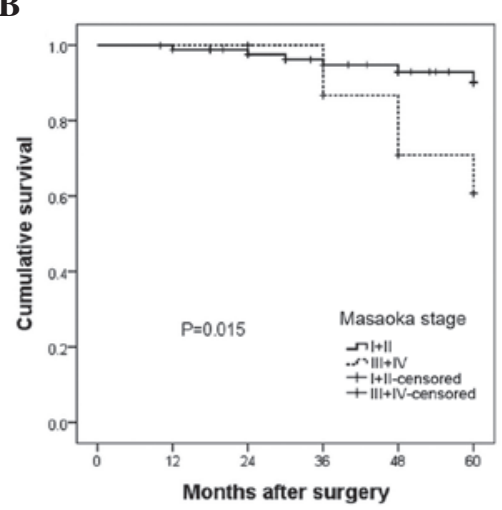

C

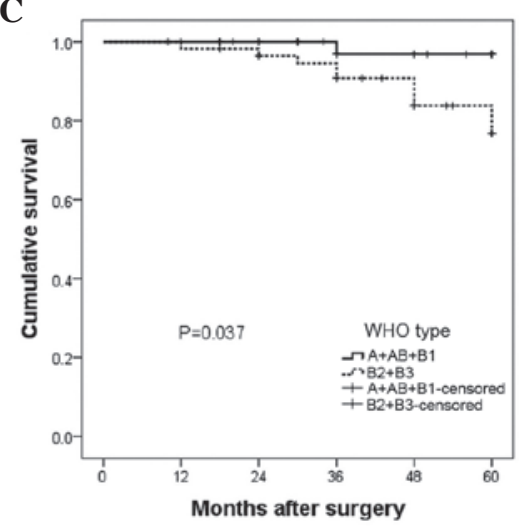

Figure 2. Overall survival curves following surgery according to MG diagnosis, Masaoka stage and WHO histological classification. (A) The overall survival rates in patients with and without MG; (B) the overall survival rates in patients according to Masaoka stage; (C) the overall survival rates in patients according to WHO histological classification. MG, myasthenia gravis; WHO, World Health Organization.

or partial superior vena cava $(n=2)$, was also performed if required. There were no perioperative mortalities, but in 7 patients perioperative myasthenic crisis occurred in the group with MG. In addition, 18 patients (17.3\%) had postoperative complications, including 10 patients with
MG that experienced assisted breathing with a ventilator due to myasthenic crisis. In addition, 4 patients contracted pneumonia, 2 had pulmonary embolisms and 2 had surgical wound infections. There were 11 mortalities during the 5-year follow-up period. The mortality-associated causes consisted 
of recurrence of thymoma in 3 patients and respiratory failure due to $\mathrm{MG}$ in 8 patients. The mortality rate in patients with MG was significantly higher compared with patients without MG $(\mathrm{P}=0.003)$.

OS rates. The OS rate at 5 years was 89.1 and $76.0 \%$ in patients without $\mathrm{MG}$ and with $\mathrm{MG}$, respectively (Fig. 2A). The OS rate was 90.0 and $68.0 \%$ for patients with Masaoka stages I + II and III + IV, respectively (Fig. 2B). The OS rate was 96.9 and $76.8 \%$ in $\mathrm{WHO}$ types $\mathrm{A}+\mathrm{AB}+\mathrm{B} 1$ and $\mathrm{B} 2+\mathrm{B} 3$, respectively (Fig. 2C). Therefore, patients with $\mathrm{MG}(\mathrm{P}=0.026)$, stages III + IV $(\mathrm{P}=0.015)$ and WHO type $\mathrm{B} 2+\mathrm{B} 3(\mathrm{P}=0.037)$ had a poorer prognosis compared with patients without these characteristics.

To determine the factors that affect patient outcome, Cox regression analysis was performed (Table III). Univariate analysis revealed that age $(\mathrm{P}=0.001)$, tumor size $(\mathrm{P}<0.001)$, MG association $(\mathrm{P}=0.042)$, WHO type $(\mathrm{P}=0.046)$, Masaoka stage $(\mathrm{P}=0.016)$ and surgical procedure $(\mathrm{P}=0.003)$ significantly affected the OS rate of patients. Multivariate analysis revealed that age $(\mathrm{P}=0.013)$ and $\mathrm{MG}$ association $(\mathrm{P}=0.037)$ significantly affected OS rate of patients.

\section{Discussion}

Although, thymomas are the most common adult tumors in the anterior mediastinal compartment, it is a rare neoplasm with an overall incidence of 0.13 cases per 100,000 individuals per year (13-15). A recent study based on a large cohort of patients with thymomas revealed that the 10-year OS rate was 0.73 (95\% confidence interval 0.69-0.75) (16). The same study reported that Masaoka stages III + IV, incomplete resection and non-thymoma histology (a diagnosis of thymic carcinoma or neuroendocrine thymic tumors) had a significant affect in increasing recurrence and in worsening survival rates of patients (16). Therefore, although the OS rate was relatively high, a cure for advanced thymomas remains a challenge $(17,18)$. Surgical resection is considered as the primary treatment of thymoma, with a reported operative mortality of $2 \%$ and a complication rate of $20 \%$ (5). Complications may include blood vessel damage, postoperative myasthenic crisis and pain, and wound infection (5). Although the role of surgical resection in the treatment of thymoma is clear, the factors that affect the outcome of patients following surgery are not fully determined. MG is the most commonly associated paraneoplastic disease in thymoma patients, but the affects of MG post-operatively remain unclear. The present study compared the outcome of patients with or without $\mathrm{MG}$ following surgery. The present results clearly demonstrated that MG is an important factor that should be considered in the management of patients with thymoma.

Thymomas usually occur in the sixth decade of life and have no significant gender predilection (19-22). In the present study, the median age of the patients was 54.6 years, which was 5 years younger than in other studies (19). The ratio of men to women in the present study was $1 / 1.4$, which was within the range of results from other studies $(19,20)$. Masaoka stages are characterized by the degree of invasion by the tumor through the capsule into surrounding tissue structures, and is an important prognostic factor in determining the most beneficial therapeutic method for a patient (11). In the 104 thymoma patients in the present study, there were $16(15.4 \%)$ patients with Masaoka stage III + IV. The patients all had successful surgical resection of the entire thymus, mediastinal fat tissue between the two phrenic nerves and adjacent organs, including the lung $(n=6)$, pericardium $(n=5)$, left brachiocephalic $(n=3)$ and partial superior vena cava $(n=2)$. However, there remained 3 recurrent cases at stage III $(3 / 14 ; 21.4 \%)$. Detterbeck (23) reported that the average recurrence rate at stage III was $30 \%$, which is higher compared with the present results. The average tumor size increased with the elevated Masaoka stage in the present study, and 5-year OS rates were 90.0 and $68.0 \%$ at stages I + II and stages III + IV, respectively, and the survival rate at stages III + IV was slightly lower compared with stages I + II.

MG occurs in 15-60\% of patients with thymoma, according to various studies (24-26). In the present results, thymoma patients with MG accounted for $36.5 \%$ of the patients. In the majority of studies, the incidence of MG in thymoma patients is the highest in WHO type B2 $(21,27,28)$. In the present study, the incidence of $\mathrm{MG}$ in type $\mathrm{A}, \mathrm{AB}, \mathrm{B} 1, \mathrm{~B} 2$ and $\mathrm{B} 3$ was 2.6, $13.2,13.2,52.6$ and $18.4 \%$, respectively. The prevalence of MG was significantly different in type B2 thymoma compared with that in other types of thymoma. In the 38 patients that had thymoma with MG, muscle weakness relief following surgery was $77.8 \%$, which is similar to the $81.1 \%$ reported by Yu et al (29). However, 1 out of 66 (1.5\%) patients without preoperative MG developed postoperative MG in the present study. This percentage was significantly lower compared with the $4.8 \%$ reported by Sun et al (30). During the surgery that the present patients underwent, ET was performed and the ectopic thymus was removed. In the MG group, the paraneoplastic thymus in $31.5 \%$ patients was hyperplastic, while it was only hyperplastic in $6.1 \%$ of patients in the group without MG. This suggests that $\mathrm{MG}$ development does not always result from thymoma. Therefore, the paraneoplastic thymus or ectopic thymic tissue may be important in MG development, which should be addressed in future studies.

There is controversy regarding whether or not MG affects the prognosis of thymomas $(26,31)$. This debate is critical to guide future management of patients with thymoma. In the present study, survival analysis revealed that the 5-year survival rate of thymoma patients with MG (76.0\%) was reduced compared with patients without MG $(89.1 \%)$ $(\mathrm{P}=0.026)$. In addition, multivariate analysis by Cox regression demonstrated that $\mathrm{MG}(\mathrm{P}=0.042)$ and age $(\mathrm{P}=0.032)$ were independent prognostic factors for survival. However, in a previous study performed in 228 patients, it was revealed that the prognosis was similar between patients with $(90.0 \%)$ and without MG (89.3\%) (31). The variance in the present and previous results are probably due to varying severities of MG, and different Masaoka stage, diagnosis methods and treatment procedures.

In summary, the present results clearly demonstrate that ET is a reliable method for the treatment of thymoma. Similarly to previous reports, the incidence of MG was significantly different in WHO type B2 thymoma compared with other types of thymoma. In addition, it is also possible that MG in certain thymoma patients was not caused by thymoma, but by the paraneoplastic thymus. Long-term survival may be expected not only for patients at early Masaoka stages, but also 
for patients without MG, and the prognosis of patients with thymoma with MG is poorer compared with patients without MG. Therefore, the present findings provide useful information for the future management of patients with thymomas.

\section{References}

1. Ruffini E and Venuta F: Management of thymic tumors: A European perspective. J Thorac Dis 6 (Suppl 2): S228-S237, 2014

2. Engels EA: Epidemiology of thymoma and associated malignancies. J Thorac Oncol 5 (10 Suppl 4): S260-S265, 2010.

3. Baas P and Rhodius R: Thymoma update 2011. Eur J Cancer 47 (Suppl 3): S315-S316, 2011.

4. Koppitz H, Rockstroh JK, Schüller H, Standop J, Skowasch D, Müller-Hermelink HK and Schmidt-Wolf IG: State-of-the-art classification and multimodality treatment of malignant thymoma. Cancer Treat Rev 38: 540-548, 2012.

5. Detterbeck FC and Parsons AM: Management of stage I and II thymoma. Thorac Surg Clin 21: 59-67, vi-vii, 2011.

6. Kaiser LR: Surgical treatment of thymic epithelial neoplasms. Hematol Oncol Clin North Am 22: 475-488, 2008

7. Suster S and Moran CA: Thymoma classification: Current status and future trends. Am J Clin Pathol 125: 542-554, 2006.

8. Thomas CR, Wright CD and Loehrer PJ: Thymoma: State of the art. J Clin Oncol 17: 2280-2289, 1999.

9. Fujii Y: Thymus, thymoma and myasthenia gravis. Surg Today 43: 461-466, 2013

10. Regnard JF, Magdeleinat P, Dromer C, Dulmet E, de Montpreville V, Levi JF and Levasseur P: Prognostic factors and long-term results after thymoma resection: A series of 307 patients. J Thorac Cardiovasc Surg 112: 376-384, 1996.

11. Masaoka A: Staging system of thymoma. J Thorac Oncol 5 (10 Suppl 4): S304-S312, 2010.

12. Ströbel P, Marx A, Zettl A and Müller-Hermelink HK: Thymoma and thymic carcinoma: An update of the WHO Classification 2004. Surg Today 35: 805-811, 2005.

13. Bushan K, Sharma S and Verma H: A review of thymic tumors. Indian J Surg Oncol 4: 112-116, 2013.

14. Kelly RJ: Thymoma versus thymic carcinoma: Differences in biology impacting treatment. J Natl Compr Canc Netw 11: 577-583, 2013.

15. Lamarca A, Moreno V andFeliu J: Thymoma and thymic carcinoma in the target therapies era. Cancer Treat Rev 39: 413-420, 2013.

16. Ruffini E, Detterbeck F, Van Raemdonck D, Rocco G, Thomas P, Weder W, Brunelli A, Evangelista A and Venuta F; European Association of Thoracic Surgeons (ESTS) Thymic Working Group: Tumours of the thymus: A cohort study of prognostic factors from the European Society of Thoracic Surgeons database. Eur J Cardiothorac Surg 46: 361-368, 2014.

17. Lucchi M and Mussi A: Surgical treatment of recurrent thymomas. J Thorac Oncol 5 (10 Suppl 4): S348-S351, 2010.

18. Riely GJ and Huang J: Induction therapy for locally advanced thymoma. J Thorac Oncol 5 (10 Suppl 4): S323-S326, 2010.

19. Gadalla SM, Rajan A,Pfeiffer R, Kristinsson SY,Björkholm M, Landgren $\mathrm{O}$ and Giaccone G: A population-based assessment of mortality and morbidity patterns among patients with thymoma. Int J Cancer 128: 2688-2694, 2011.
20. Nakagawa K, Asamura H, Matsuno Y, Suzuki K, Kondo H, Maeshima A, Miyaoka E and Tsuchiya R: Thymoma: A clinicopathologic study based on the new World Health Organization classification. J Thorac Cardiovasc Surg 126: 1134-1140, 2003.

21. Patel S, Macdonald OK, Nagda S, Bittner N and Suntharalingam M: Evaluation of the role of radiation therapy in the management of malignant thymoma. Int J Radiat Oncol Biol Phys 82: 1797-1801, 2012.

22. Rena O, Papalia E, Oliaro A, Ruffini E, Filosso P, Novero D, Maggi $G$ and Casadio $C$ : Does adjuvant radiation therapy improve disease-free survival in completely resected Masaoka stage II thymoma? Eur J Cardiothorac Surg 31: 109-113, 2007.

23. Detterbeck FC: Evaluation and treatment of stage I and II thymoma. J Thorac Oncol 5 (10 Suppl 4): S318-S322, 2010.

24. Kondo K and Monden Y: Thymoma and myasthenia gravis: A clinical study of 1,089 patients from Japan. Ann Thorac Surg 79: 219-224, 2005.

25. López-Cano M, Ponseti-Bosch JM, Espin-Basany E, Sánchez-Garcia JL and Armengol-Carrasco M: Clinical and pathologic predictors of outcome in thymoma-associated myasthenia gravis. Ann Thorac Surg 76: 1643-1649; disc 1649, 2003.

26. Margaritora S, Cesario A, Cusumano G, Meacci E, D'Angelillo R, Bonassi S, Carnassale G, Porziella V, Tessitore A, Vita ML, et al: Thirty-five-year follow-up analysis of clinical and pathologic outcomes of thymoma surgery. Ann Thorac Surg 89: 245-252; discussion 252, 2010.

27. Kondo K, Yoshizawa K, Tsuyuguchi M, Kimura S Sumitomo M, Morita J, Miyoshi T, Sakiyama S, Mukai K and Monden Y: WHO histologic classification is a prognostic indicator in thymoma. Ann Thorac Surg 77: 1183-1188, 2004.

28. Okumura M, Ohta M, Tateyama H, Nakagawa K, Matsumura A, Maeda H, Tada H, Eimoto T, Matsuda $\mathrm{H}$ and Masaoka A: The World Health Organization histologic classification system reflects the oncologic behavior of thymoma: A clinical study of 273 patients. Cancer 94: 624-632, 2002.

29. Yu S, Li F, Chen B, Lin J, Yang M, Fu X, Li J and Bu B: Eight-year follow-up of patients with myasthenia gravis after thymectomy. Acta Neurol Scand 131: 94-101, 2015.

30. Sun XG, Wang YL, Liu YH, Zhang N, Yin XL and Zhang WJ: Myasthenia gravis appearing after thymectomy. J Clin Neurosci 18: 57-60, 2011.

31. Yu L, Zhang XJ, Ma S, Jing Y, Li F and Krasna MJ: Different characteristics of thymomas with and without myasthenia gravis. Ann Surg Oncol 19: 94-98, 2012. 